Résumés des conférences et travaux

\title{
Langue et civilisation méroïtiques
}

Claude Rilly

\section{(2) OpenEdition \\ Journals}

Édition électronique

URL : https://journals.openedition.org/ashp/4079

DOI : $10.4000 /$ ashp.4079

ISSN : 1969-6310

Éditeur

Publications de l'École Pratique des Hautes Études

Édition imprimée

Date de publication : 1 septembre 2021

Pagination : 23-30

ISSN : 0766-0677

Référence électronique

Claude Rilly, "Langue et civilisation méroïtiques », Annuaire de l'École pratique des hautes études (EPHE), Section des sciences historiques et philologiques [En ligne], 152 | 2021, mis en ligne le 14 juin 2021, consulté le 14 juin 2022. URL : http://journals.openedition.org/ashp/4079 ; DOI : https://doi.org/ 10.4000/ashp.4079 


\title{
LANGUE ET CIVILISATION MÉROÏTIQUES
}

\author{
Directeur d'études : M. Claude RILLY
}

Programme de l'année 2019-2020 : I. Introduction à l'histoire des royaumes du Soudan ancien. - II. Initiation à l'écriture et à la langue méroïtiques. - III. Études des textes funéraires méroïtiques de Sedeinga.

La direction d'études cumulante «Langue et civilisation méroïtique » a été créée à l'EPHE, section des Sciences historiques et philologiques en 2019. Le présent rapport couvre donc la première année de ce nouvel enseignement. Nous voudrions ici remercier nos collègues égyptologues, Pascal Vernus et Michel Chauveau, pour avoir suggéré cette création et Andréas Stauder pour l'avoir ardemment promue et en avoir finalisé les modalités avec les autorités de notre École.

L'enseignement de la langue de Méroé n'est toutefois pas nouveau à l'EPHE. De 1964 à 1990, Jean Leclant (1920-2011) a en effet assuré à la section des Sciences religieuses une direction d'études intitulée « Histoire de la diffusion des cultes égyptiens » qui comportait deux thèmes, d'une part les « Recherches sur la diffusion des cultes isiaques » et d'autre part les «Études méroïtiques ». Bien qu'axé originellement sur «les textes funéraires méroïtiques »- son intitulé de 1964 à 1966 -, le second cours a très rapidement inclus tous les sujets philologiques et linguistiques liés à la langue méroïtique. C'est en grande partie là que s'est poursuivi le projet lancé en 1958 avec la collaboration d'André Heyler (1924-1971), le Répertoire d'épigraphie méroïtique (REM) ${ }^{1}$. Rassemblant tous les textes dont les publications étaient dispersées dans d'innombrables revues et monographies, cet ouvrage visait à établir un corpus exhaustif des inscriptions méroïtiques. En 1990, Jean-Claude Grenier (1943-2012) a succédé à Jean Leclant sur une chaire désormais intitulée « Religion égyptienne dans les mondes hellénistique et romain », qui ne reprenait donc pas la composante méroïtique de la direction d'études originelle. Enfin, après une période de latence de quelques années, cet enseignement, toujours exclusivement centré sur sa composante égyptienne, a été confié en 2018 à Ivan Guermeur dans le cadre d'une chaire non cumulante.

L'étude du méroïtique est donc passée, après une longue absence de trente ans, de la $V^{e}$ section à la $I^{e}$, ce qui est, convenons-en, sa place naturelle au côté d'autres idiomes disparus. La continuité entre ces deux enseignements est d'autant plus étroite que le professeur Leclant m'avait, en 2001, confié la responsabilité de son dossier méroïtique ${ }^{2}$ et, en 2008 , celle de la mission archéologique qu'il avait dirigé à Sedeinga, en Nubie soudanaise, un site qui a livré et continue de livrer de nombreuses

1. J. Leclant, A. Heyler (†), C. Berger-El-Naggar, C. Carrier, C. Rilly, Répertoire d'épigraphie méroïtique. Corpus des inscriptions publiées, vol. I, II, III, Paris, Académie des inscriptions et belles-lettres, 2000.

2. C. Rilly, «Les études méroïtiques », dans Au fil du Nil, le parcours d'un égyptologue : Jean Leclant. Colloque de la fondation Singer-Polignac, Paris, 12 novembre 2001, Paris, Académie des inscriptions et belles-lettres, 2002, p. 95-105, fig. 59-67. 
inscriptions méroïtiques ${ }^{3}$. L'EPHE peut ainsi s'enorgueillir de continuer à offrir le seul enseignement au monde consacré à cette langue, la plus anciennement écrite en Afrique subsaharienne. Une initiation aux textes méroïtiques a bien existé dans le cadre des chaires d'archéologie soudanaise, par Fritz Hintze (1925-1993) à l'université Humboldt de Berlin, Nicolas B. Millet (1934-2004) à l'université de Toronto, Inge Hofmann (1939-2016) à l'université de Vienne, mais seule l'EPHE a assuré et continue à assurer un enseignement universitaire complet de cette discipline.

Dans l'intervalle de trente années qui sépare l'enseignement de Jean Leclant de cette nouvelle direction d'études, le lent déchiffrement du méroïtique s'est poursuivi avec des hauts et des bas. On rappelle que l'écriture a été déchiffrée en 1911 par l'égyptologue britannique Francis Llewellyn Griffith ${ }^{4}$, mais que la langue, disparue sans descendance, est incomplètement traduite, à l'instar de l'étrusque, du gaulois et de beaucoup d'autres idiomes antiques. De 1991, date de la dernière monographie d'Inge Hofmann ${ }^{5}$ à 1999, où parut mon premier article sur la lecture d'un signe méroïtique $^{6}$, aucune étude philologique ou linguistique notable ne fut publiée. Le découragement le plus complet devant la lenteur des progrès avait gagné la communauté sans cesse plus étroite des méroïtisants. Dans son dernier rapport de l'Annuaire de l'EPHE en 19897, Jean Leclant écrivait ainsi : «Attendu que la nature profonde de la langue méroïtique échappe encore, le seul espoir semble devoir être placé dans la découverte d'une inscription bilingue. » On sait toutefois que cet espoir est bien mince et qu'un seul bilingue, s'il peut permettre le déchiffrement d'une écriture, ne saurait assurer la compréhension intégrale d'une langue.

D'autres pistes existaient, à commencer par un examen complet de ce qui avait écrit sur le sujet dans de nombreuses publications dispersées. Cette synthèse fut l'objet de mon « diplôme de l'EPHE » en 2001 et fut publiée dans les collections de l'École pratique des hautes études en $2007^{\circ}$. Elle a permis en outre des progrès dans plusieurs domaines, notamment la paléographie, la phonologie ainsi que la syntaxe du groupe nominal et des phrases non verbales. Toutefois, l'avancée la plus prometteuse a été l'identification de la famille linguistique du méroïtique, une question ardemment débattue depuis près d'un siècle. Présentée dans ma thèse de doctorat de l'EPHE sous la direction de Pascal Vernus en 2003, elle fut publiée en $2010^{9}$. Le méroïtique appartient, comme il avait déjà été pressenti ${ }^{10}$, mais jamais prouvé de manière satisfaisante, à un sous-groupe du phylum nilo-saharien que j'ai nommé «soudanique

3. C. Rilly, « La mission archéologique française de Sedeinga. De la reine Tiyi aux dames de Nubie », dans M. Maillot (éd.) SFDAS. Section française de la Direction des antiquités du Soudan, 1969-2019, Paris, Soleb, Bleu Autour, 2020, p. 27-49.

4. F. Ll. Griffith, The Meroitic Inscriptions of Shablûl and Karanóg, Philadelphie, 1911.

5. I. Hofmann, Steine für die Ewigkeit / Meroitische Opfertafeln und Totenstelen, Beiträge zur Sudanforschung, Vienne, 1991 (Beiheft 6).

6. «Une nouvelle lecture du signe méroïtique Q. », Göttinger Miszellen, 169 (1999), p. 101-110.

7. J. Leclant. «Conférence de M. Jean Leclant», dans École pratique des hautes études. Section des sciences religieuses. Annuaire, t. 98, 1989-1990, Paris, 1989, p. 202-212.

8. C. Rilly, La langue du royaume de Méroé, Paris, Champion, 2007 (Bibliothèque de l'École des hautes études. Sciences historiques et philologiques, 344).

9. C. Rilly, Le méroïtique et sa famille linguistique, Paris, Louvain, Peeters, 2010 (Afrique et Langage, 14).

10. B. G. Trigger, « Meroitic and Eastern Sudanic: a Linguistic Relationship? », Kush, 12 (1964), p. 188194. 
oriental nord » et qui rassemble les parlers nubiens actuels d'Égypte et du Soudan, le nara d'Érythrée, les langues taman du Darfour et du Ouaddaï et le groupe nyima des monts Nouba. Cette découverte a permis de nombreuses avancées, notamment dans la traduction du lexique et, récemment, dans l'identification des pronoms personnels ${ }^{11}$. En raison toutefois de la profondeur chronologique qui sépare les différents groupes du soudanique oriental nord, comparable à celle de l'indo-européen, les langues apparentées au méroïtique n'en sont pas suffisamment proches pour permettre de le traduire facilement. On doit souvent recourir à la reconstruction de proto-formes et on ne peut toujours pas se passer des méthodes d'analyse interne qui étaient les seules possibles jusqu'ici. La tâche est encore immense avant de pouvoir traduire de façon satisfaisante les inscriptions les plus obscures, notamment les chroniques royales, mais il n'est plus possible d'écrire, comme on le lit encore trop souvent dans les ouvrages de vulgarisation, que le méroïtique est une langue inconnue ou mystérieuse.

Si l'étude linguistique sur la langue de Méroé a été, durant ces deux dernières décennies, presque entièrement l'œuvre de l'auteur de ces lignes, on doit saluer le travail impressionnant de l'égyptologue allemand Jochen Hallof dans la publication de 779 textes méroïtiques inédits retrouvés dans les fouilles britanniques de Qasr Ibrim, en Nubie égyptienne ${ }^{12}$. Signalons également que deux thèses de doctorat, l'une sur la phonologie du méroïtique par Kirsty Rowan (School of Oriental and African Studies, Londres), l'autre sur les textes royaux par Gilda Ferrandino (université L'Orientale, Naples), ont été présentées respectivement en 2007 et 2016. Cette dernière est en cours de publication.

Le programme de cette première année d'enseignement avait été initialement conçu pour une série de deux cours hebdomadaires d'une heure chacun : $1^{\text {er }}$ semestre, $1^{\text {re }}$ heure : introduction à l'histoire des royaumes du Soudan ancien; $2^{\mathrm{e}}$ heure : initiation à l'écriture et à la langue méroïtiques. $2^{\mathrm{e}}$ semestre, $1^{\text {re }}$ heure : initiation à l'écriture et à la langue méroïtiques; $2^{\mathrm{e}}$ heure : étude des textes funéraires méroïtiques de Sedeinga.

La pandémie de Covid-19 a bouleversé ce programme. Les cours ont pu s'effectuer en présence physique jusqu'au 10 mars dans les locaux du boulevard Raspail. Toutefois, j'ai été absent du 11 novembre au 15 décembre pour diriger la mission archéologique annuelle de Sedeinga. Une heure de cours supplémentaire à partir de mars 2020 a donc été programmée pour rattraper cette absence. Mais le 16 mars, le gouvernement a fermé les universités jusqu'à nouvel ordre. Après avoir consulté mes 21 élèves, il s'est avéré que nombre d'entre eux seraient dispersés dans des localités de province et que beaucoup n'auraient pas une connexion suffisante à Internet pour suivre un enseignement par visio-conférence. La suite des cours du second semestre s'est donc effectuée par échange d'e-mails dans un premier temps. J'ai envoyé du 19 mars au 11 mai un cours complet chaque semaine, comprenant leçon et exercice, ainsi qu'un article scientifique à lire et j'ai répondu par le même moyen aux questions des élèves. Par la suite, j'ai enregistré chaque semaine une conférence sur une chaîne YouTube créée pour la circonstance et nommée « EPHE méroïtique » ${ }^{13}$.

11. C. Rilly, «Personal Markers and Verbal Number in Meroitic », Dotawo, 7 (2020), consultable en ligne à l'adresse https://pages.sandpoints.org/dotawo/article/rilly/.

12. J. Hallof, The Meroitic inscriptions from Qasr Ibrim, Dettelbach, Röll Verlag, 7 vol., 2011-2020.

13. https://www.youtube.com/channel/UCDRcGetYHm8RSHnHBZrnc-Q. Les exigences de ce medium ont obligé à sectionner chaque conférence en plusieurs volets. 


\section{Conférence inaugurale}

Pour marquer le retour des études méroïtiques à l'EPHE, la première conférence a eu lieu le 15 octobre 2019, sur invitation, à la salle d'égyptologie de Paris IV-Sorbonne, gracieusement mise à notre disposition par le professeur Pierre Tallet ${ }^{14}$. Après un bref rappel de l'histoire des royaumes de Kouch et des principales avancées dans le décryptage de la langue méroïtique depuis le déchiffrement de l'écriture par Griffith, trois études ont été présentées : paléographie et cohérence du système graphique, deixis et déchiffrement, identification contextuelle et méthode comparative. La première constituait une recherche inédite sur les réformes orthographiques qui ont marqué le milieu du règne conjoint de la Candace Amanitoré et de son fils le roi Natakamani (vers 60 apr. J.-C.). La seconde montrait comment, grâce à l'identification du morphème qo comme adjectif et pronom démonstratif, son occurrence dans les légendes iconographiques permettait de relier les substantifs qu'il accompagne aux images qu'il commente, livrant ainsi le nom de divers animaux comme le chien (wle), le faucon (kele ou kere), la gazelle (abese) et le serpent (pete), autant de mots qui présentent des cognats dans les langues apparentées, particulièrement en nubien. Enfin, la troisième étude détaillait la traduction du mot pwrite " vie », « force vitale », équivalent méroïtique de l'égyptien ' $n h$, premièrement par le moyen de parallèles égyptiens, puis par la recherche de cognats dans les langues apparentées, notamment le nubien et le nara.

\section{Histoire des royaumes du Soudan ancien}

Il était indispensable, en cette première année d'enseignement de la langue et de la civilisation méroïtiques, de présenter l'histoire du pays de Kouch depuis la fin du néolithique (3500 av. J.-C.) jusqu'à la chute du royaume de Méroé (330 apr. J.-C.). Elle est en effet souvent méconnue, y compris des égyptologues qui constituent une partie importante de nos élèves. Il était originellement prévu d'y consacrer une heure par semaine sur la durée du premier semestre. Cette prévision s'est avérée beaucoup trop optimiste. Une présentation détaillée de l'histoire kouchite, incluant une discussion sur des points récemment découverts ou actuellement débattus et abondamment illustrée au moyen d'un Powerpoint par cours, exige une trentaine d'heures. Cette présentation ne s'achèvera donc que lors de la prochaine année universitaire.

Les cours en présence physique ont permis de traiter le Pré-Kerma (3500-2500 av. J.-C.), le royaume de Kerma (2500-1450 av. J.-C.), la colonisation égyptienne de la Basse-Nubie au Moyen Empire (2000-1650 av. J.-C.), la colonisation égyptienne de Kouch au Nouvel Empire (1450-ca 900 av. J.-C.), la première royauté de Napata ( ca 900-715 av. J.-C.), la XXV dynastie kouchite (715-664 av. J.-C.) et la première partie du second royaume de Napata (664-593 av. J.-C.). Durant les cours par correspondance informatique, faute de pouvoir avancer avec l'ensemble des étudiants, l'histoire de toutes ces périodes a été révisée et consolidée.

14. Cette conférence inaugurale a été enregistrée en 10 volets sur YouTube : https://www.youtube.com/ watch? $\mathrm{v}=5 \mathrm{~V}$ sKD--bHPE\& $\mathrm{t}=479 \mathrm{~s}$. 
Ce panorama historique s'est appuyé sur l'étude de textes égyptiens en translittération et traduction, à savoir l'autobiographie de Herkhouf ${ }^{15}$ et la chronique de la tombe de Sobeknakht à El-Kab ${ }^{16}$ pour le royaume de Kerma, les inscriptions d'exécration de Mirgissa ${ }^{17}$ pour la colonisation égyptienne au Moyen Empire, l'autobiographie d'Ahmosé fils d'Abana ${ }^{18}$ pour la colonisation égyptienne au Nouvel Empire, la stèle de grès de Piânkhy (Khartoum $\left.n^{\circ} 1851\right)^{19}$ et sa stèle triomphale de l'an $21^{20}$ pour le premier royaume de Napata, la Pierre de Shabaqo du British Museum ${ }^{21}$, les stèles Kawa IV et VI de Taharqo ${ }^{22}$ et la stèle du songe de Tanouétamani ${ }^{23}$ pour la $\mathrm{XXV}^{\mathrm{e}}$ dynastie, la stèle Kawa VII d'Anlamani ${ }^{24}$ et la stèle de l'élection d'Aspelta (Caire JE 48866) ${ }^{25}$ pour la première période du second royaume de Napata.

On a particulièrement examiné des problématiques récentes ou encore irrésolues. Pour le royaume de Kerma, l'hypothèse de Karola Zibelius-Chen ${ }^{26}$, qui attribue à un remplacement d'élite le changement de nom de Kerma, passant de Yam (J3m / Jm3) à Kouch ( $K 3 z$ dans la stèle de Florence) dans les textes égyptiens, respectivement de la $V^{e}$ et de la XII ${ }^{e}$ dynastie, a semblé judicieuse. Elle explique en effet, d'une part la continuité culturelle entre le pré-Kerma et le Kerma ancien et d'autre part l'existence d'une élite de langue proto-méroïtique au Kerma moyen et classique, attestée par les anthroponymes locaux transcrits en hiératique. Ce serait donc durant la Première Période intermédiaire qu'un basculement se serait produit, permettant à une population issue de l'Ouest du Soudan de prendre le pouvoir dans le royaume de Kerma. Pour les origines du premier royaume de Napata, c'est-à-dire la chronologie des tombes anciennes de la nécropole royale d'el-Kurru, il n'est toujours pas possible de se prononcer entre l'interprétation de L. Török (chronologie longue, démarrant

15. Pour une édition commentée récente de ce texte, voir C. Obsomer, «Les expéditions d'Herkhouf (VI ${ }^{\mathrm{e}}$ dynastie) et la localisation de Iam », dans M.-C. Bruwier, Pharaons noirs. Sur la piste des Quarante jours, Musée royal de Mariemont, 2007, p. 39-52.

16. Voir W. V. Davies, « Kush in Egypt », Sudan \& Nubia, 7 (2003), p. 52-55.

17. Y. Koenig, «Les textes d'envoûtement de Mirgissa », Revue d'Égyptologie, 41 (1990), p. 101-125.

18. Voir C. Vandersleyen, Les guerres d'Amosis, fondateur de la XVIII ${ }^{e}$ dynastie, Bruxelles, 1971 (Monographies Reine Élisabeth), p. 17-87.

19. FHN I = T. Eide, T. Hägg, R.-H. Pierce, L. Török, Fontes Historiae Nubiorum: I From the 8th Century to the 6th B.C. Textual Sources for the History of the Middle Nile Between the 8th Century B.C. and the 6th A.D., Bergen, University of Bergen, 1994, p. 55-62; F. Colin, « Le faiseur de rois et de chefs libyens, sur la stèle de Napata au Musée de Khartoum, SNM 1851 », Carnet de laboratoire en archéologie égyptienne, 6 mai 2020, § 1-30, https://clae.hypotheses.org/189.

20. N. Grimal, Études sur la propagande royale égyptienne, I. La stèle triomphale de Pi('ankh)y au musée $d u$ Caire JE 48862 et 47086-47089, Le Caire; FHN I (voir n. 19), p. 62-119.

21. Pour une étude récente, voir Joshua J. Bodine, « The Shabaka Stone: An Introduction », Studia Antiqua, 7, $\mathrm{n}^{\circ} 1$ (2009) https://scholarsarchive.byu.edu/studiaantiqua/vol7/iss1/3.

22. FHN I, p. 135-145 et 164-176.

23. N. Grimal, Quatre stèles napatéennes au Musée du Caire JE 48863-4886, IFAO, Le Caire, 1981, p. 3-20; FHN I, p. 193-209.

24. FHN I, p. 216-228.

25. FHN I, p. $230-252$.

26. K. Zibelius-Chen, « Sprachen Nubiens in pharaonischer Zeit. », Lingua Aegyptia, 22 (2014), p. $267-$ 309. 
vers 995 av. J.-C.) ${ }^{27}$ et celle de T. Kendall reprenant les conclusions de Reisner (chronologie courte démarrant vers 890 av. J.-C. $)^{28}$. Les récentes fouilles de Tombos et Amara Ouest ont montré une continuité d'occupation des villes de colonisation égyptienne après la fin du Nouvel Empire, mais on ignore encore de quel pouvoir politique elles dépendaient. On a également examiné de près les arguments en faveur d'une inversion de la succession traditionnellement admise Shabaqo - Shabataqo au sein de la $X X X V^{e}$ dynastie. Proposée originellement par Michael Bányai, elle a été reprise par plusieurs spécialistes et étudiée en détail en 2014 par Frédéric Payraudeau ${ }^{29}$. Cette proposition, que j'avais dans mon Histoire du Soudan considérée comme une simple hypothèse ${ }^{30}$, semble désormais bien établie et repose sur des bases solides, notamment les concordances entre les années régnales des pharaons de la XXVe dynastie et des souverains assyriens.

\section{Initiation à l'écriture et à la langue méroïtique}

En cette première année d'enseignement, l'étude de la langue de Kouch, plus encore que son histoire, était pour les étudiants une terra incognita. Il a donc été nécessaire de procéder à une initiation progressive, quasi-scolaire, comprenant des exercices et leur correction. Il ne sera possible que l'an prochain d'introduire dans ce cours l'activité de recherche qui fait la spécificité de l'École pratique des hautes études.

L'apprentissage des deux écritures, la cursive et l'hiéroglyphique, a occupé les trois premiers cours. On est passé de textes rédigés en police de caractères méroïtiques à des fac-similés, puis à des photographies de textes réels. Les différentes périodes paléographiques ont fait l'objet d'un cours spécifique, montrant l'évolution importante des ductus de certains signes entre les inscriptions les plus anciennes (Archaïque $\mathrm{A}, \mathrm{III}^{\mathrm{e}}$ siècle av. J.-C.) et les plus récentes (Tardif $\mathrm{B}, \mathrm{V}^{\mathrm{e}}$ siècle de notre ère).

Pour les études d'inscriptions, on a privilégié les textes funéraires. À l'exception des titres administratifs ou sacerdotaux, qui pour la plupart restent obscurs, ces longues épitaphes sont désormais presque entièrement traduisibles.

Les textes suivants ont été lus, traduits et commentés :

- REM 0261, stèle de la nécropole de Karanog;

- REM 1068, table d'offrandes de la nécropole de Qustul;

27. L. Török, « The Origin of the Napatan State: The Long Chronology of the El Kurru Cemetery. A contribution to T. Kendall's Main Paper », dans S. Wenig (éd.), Studien zum antiken Sudan. Akten der 7. Internationalen Tagung für meroitistische Forschungen vom 14. bis 19. September 1992 in Gosen bei Berlin, Wiesbaden, 1999 (Meroitica 15), p. 149-159.

28. T. Kendall, "The Origin of the Napatan State: El Kurru and the Evidence for the Royal Ancestors », dans S. Wenig (éd.), Studien zum antiken Sudan. Akten der 7. Internationalen Tagung für meroitistische Forschungen vom 14. bis 19. September 1992 in Gosen bei Berlin, Wiesbaden, 1999 (Meroitica 15), p. 149-159.p. 3-117.

29. M. G. Bányai, « Ein Vorschlag zur Chronologie der 25. Dynastie in Ägypten », Journal of Egyptian History, 6 (2013), p. 46-129; F. Payraudeau, « Retour sur la succession Shabaqo-Shabataqo », NeHeT, 1 (2014), p. 115-127.

30. C. Rilly, « Histoire du Soudan, des origines à la chute du sultanat Fung », dans O. Cabon (éd.), Histoire et civilisations du Soudan, Soleb, Bleu Autour, Paris, 2017 (Études d'égyptologie 15), p. 25-445. 
- REM 0264, table d'offrandes de la nécropole de Karanóg;

- REM 1091, linteau inscrit de la nécropole de Sedeinga (extraits);

- REM 0273, stèle de la nécropole de Karanog;

- Stèle funéraire de Sedeinga au nom de la dame Ataqeloula (II T 355s1) ${ }^{31}$;

- REM 0300, table d'offrandes de la nécropole de Karanog;

- REM 1057, stèle de la nécropole d'Aksha;

— REM 0108, légende iconographique de la « Chambre méroïtique » de Philae;

- REM 1089, inscription votive sur socle de statuette de la reine Nawidémak;

- REM 1273, stèle de la nécropole de l'île de Saï;

- REM 1149, stèle de la nécropole de Nag Gamus;

- REM 0251, stèle de la nécropole de Karanog;

- REM 0127, inscription archaïque sur une statuette du dieu-lion Apédémak;

- REM 0279, table d'offrandes de la nécropole de Karanog;

- Stèle funéraire de Sedeinga au nom de la dame de la dame Maliwaras (II T 410 d1).

L'initiation à la langue méroïtique a également privilégié la morphologie et les structures grammaticales qui apparaissent dans les épitaphes et qui sont désormais bien balisées. Elle a porté uniquement sur les constituants du groupe nominal : usages de l'article, construction du vocatif, des deux génitifs (antéposé et postposé), assimilation des constituants («loi de Griffith»), postpositions simples et composées, constructions concaténées en miroir (avec des parallèles en sumérien), copule au singulier et pluriel, phrases non-verbales. Ces éléments grammaticaux ont permis d'entrer dans le détail des différentes parties des épitaphes : invocation aux divinités de l'Au-delà, nomination du défunt ou de la défunte, filiation maternelle et paternelle, description de la carrière du défunt (description individuelle), mention des dignités de la parentèle (description relative).

Les bénédictions finales, c'est-à-dire les prières aux divinités, n'ont été que superficiellement étudiées cette année en raison des circonstances particulières dues à la pandémie. Elles font en effet appel à l'étude de la morphologie verbale, dont la reconstruction est un travail en cours, même si les formes verbales utilisée dans les épitaphes sont généralement traduites de manière satisfaisante. Ce sujet sera abordé l'an prochain.

\section{Les textes funéraires de Sedeinga}

L'étude des premières épitaphes découvertes sur le site de Sedeinga a commencé dès la première année d'enseignement de Jean Leclant à la Ve section, en 1964. Il était alors l'épigraphiste en titre de la mission italienne de Soleb et Sedeinga, dirigée par Michela Schiff Giorgini. Les travaux archéologiques sur Soleb étant achevés en 1976, Sedeinga devint une mission à part entière et sa concession fut cédée à la France. Jean Leclant en prit la direction, qu'il exerça jusqu'en 1991, étant alors remplacé par Audran Labrousse, puis par Catherine Berger-El Naggar. Depuis les premiers coups de pelles, le site a régulièrement fourni des inscriptions méroïtiques, une cinquantaine

31. Cette stèle, comme les autres inscriptions récemment découvertes, ne porte pas encore de numéro de REM (Répertoire d'épigraphie méroïtique, voir n. 1). 
en tout, ce qui est considérable pour une nécropole méroïtique et n'est dépassé que par les cimetières de Karanog et de Faras, en Basse-Nubie, qui étaient les sièges successifs des vice-rois de Nubie. Les dernières années ont été particulièrement fastes puisque vingt-deux textes ont été mis au jour entre 2016 et 2019, la plupart étant en excellent état de conservation et de bonne longueur.

Prévue pour le second semestre de 2020-2021, l'étude des textes de Sedeinga n'a comporté que deux cours en présence physique, consacrés à la présentation du site. Par la suite, trois inscriptions ont été lues, traduites et commentées dans les cours par correspondance, à savoir REM 1091 (linteau inscrit du prince Natemakhora découvert en 1964, extraits), II T 355s 1 (stèle de la dame Ataqeloula découverte en 2016), II T 140d1 (stèle de la dame Maliwaras découverte en 2017). 